\title{
Interference-Aware Multicasting in Wireless Mesh Networks*
}

\author{
Sudheendra Murthy ${ }^{1}$, Abhishek Goswami ${ }^{2}$, and Arunabha Sen ${ }^{1}$ \\ ${ }^{1}$ School of Computing and Informatics, Arizona State University, Tempe, AZ 85287 \\ sudhi@asu.edu, asen@asu.edu \\ 2 Mobile Devices Software, Motorola \\ abhishek.goswami@motorola.com
}

\begin{abstract}
Multicasting is one of the most important applications in Wireless Ad hoc Networks and the currently emerging Wireless Mesh Networks. In such networks, interference due to the shared wireless medium is a prime factor in determining the data rate achievable by a multicast application. In this research work, we present an interferenceaware multicast routing algorithm that takes into account the effects of interference to determine the maximum bandwidth multicast structure. We characterize the problem of computing maximum bandwidth multicast structure as a graph problem of finding minimum degree weakly induced subgraph in a graph subject to the connectivity and interference constraints. We establish the intractability of the problem and provide efficient heuristic that performs close to the optimal in most of the cases. We also present the design of a more practical distributed algorithm. The simulation results demonstrate the benefits of our heuristic over Shortest Path Tree and Minimum Steiner Tree approximation algorithms.
\end{abstract}

Keywords: Wireless Mesh Network, Minimum Interference Multicast, Weakly Induced Connected Subgraph, NP-Complete.

\section{Introduction}

Research in Wireless Mesh Networks (WMNs) has gained tremendous momentum recently as a result of its commercial deployment in many US cities including Seattle, Philadelphia, Tempe 1. WMNs are increasingly being used to provide cost effective and reliable Internet connectivity to residents and businesses in these cities. These WMNs consist of a set of wireless routers (access points) to which the end users connect, a set of wireless routers that act as forwarding nodes and a set of gateway routers that provide connectivity to the Internet. Data from the end users is routed in the WMN towards the gateway routers and to the Internet.

\footnotetext{
* This research was supported in part by ARO grant W911NF-06-1-0354. The information reported here does not reflect the position or the policy of the federal government.
} 
The widespread deployment of WMNs has fueled research work in providing better support to multimedia applications like real-time video transport and Voice over the Internet (VoIP) services. Common to many of these applications is the need for a multicast framework that facilitates efficient distribution of datagrams to a cohort of hosts. Multicasting results in bandwidth savings as compared to multiple unicast sessions. Early efforts in providing multicast support in WMNs ignored to consider the interference effects of the shared wireless medium in which the mesh routers operate. Interference is an important factor that dictates the bandwidth available for the multicast transmission. The mesh routers are usually equipped with IEEE $802.11 \mathrm{a} / \mathrm{b} / \mathrm{g}$ interfaces. Interference in the context of this paper refers to the bandwidth sharing effect between nodes operating in close range caused due to the CSMA/CA nature of 802.11. In this paper, we present a novel multicast framework that considers the effects of interference and constructs a multicast structure that provides maximum bandwidth to the applications. Our objective is to identify multicast forwarding group nodes whose transmission induces least amount of interference. To the best of our knowledge, this is the first paper that proposes such a framework. The main contributions of this work are as follows.

1. We provide a novel formulation of the interference-aware multicasting problem as a graph problem of finding weakly induced subgraph in a graph representing the mesh routers.

2. We prove the NP-completeness of the problem, thereby establishing its intractable nature.

3. We provide an Integer Linear Program technique of optimally solving this problem.

4. We provide a centralized heuristic algorithm that performs close to the optimal and describe a distributed implementation of the algorithm. We compare the performance of the proposed heuristic with the optimal, shortest path solution and minimum Steiner tree solution.

The road map for rest of the paper is as follows. In section 2, we introduce the required notation and give the formal problem definition. The complexity analysis of the problem is provided in section 3. The Integer Linear Program formulation to obtain the optimal solution is provided in section 4 . The centralized heuristic and the design of its distributed extension are provided in section 5] Section 6 presents the evaluation of our heuristics. Section 7 reviews the related work in this area, while section 8 concludes the paper.

\section{Problem Formulation}

\subsection{Wireless Transmission and Interference Model}

We assume uniform transmission range $R_{T}$ and interference range $R_{I}$ for all the routers in the WMN. We represent a WMN by a directed potential communication graph $G(V, E)$ in which the vertices represent the mesh routers. Denote 


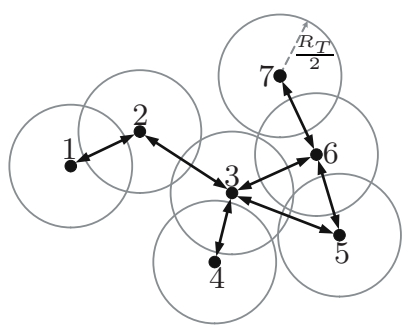

Fig. 1. A Potential Communication Graph $G(V, E)$ with transmission radius $R_{T}$

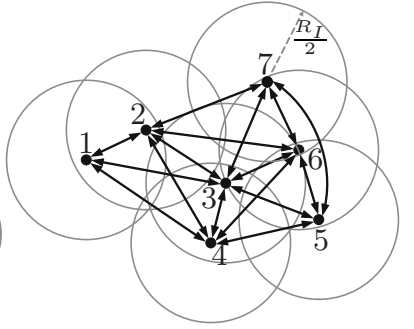

Fig. 2. Potential Interference Graph $H(V, F)$ with interference radius $R_{I}=1.5 R_{T}$

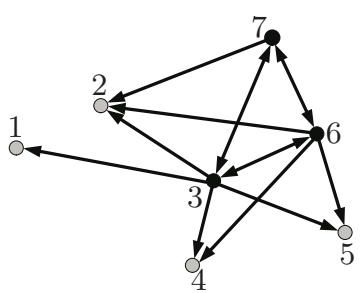

Fig. 3. $\langle S\rangle_{H}$, the subgraph weakly induced by the vertex set $S=\{3,6,7\}$ on the graph of figure 2

the Euclidean distance between routers $u$ and $v$ by $\operatorname{dist}(u, v)$. Two directed edges $\{(u, v),(v, u)\} \in E$ if $\operatorname{dist}(u, v) \leq R_{T}$ and implies that mesh router $u$ can communicate directly with mesh router $v$. We assume that each router is equipped with one radio and all routers operate on the same channel. Any of the mesh routers can be the source node in the multicast tree. However in many applications, the multicast source comes from the wired network and hence, the multicast source is a gateway mesh router. An access point router becomes a receiver of the multicast structure if there is at least one end-user connected to the access point who wants to receive this multicast stream. We assume a 802.11 CSMA/CA medium access control schemen. Thus, a transmission between two nodes may prevent all nodes within the transmission range of the sender from transmitting due to carrier sensing.

We assume that the interference range $R_{I}$ is $q \times R_{T}$ with $q \geq 1$. We model the co-channel interference of the WMN with the help of a directed potential interference graph. A directed potential interference graph is represented by $H(V, F)$ in which the vertex set corresponds to the mesh routers. Two directed edges $\{(u, v),(v, u)\} \in F$ if $\operatorname{dist}(u, v) \leq R_{I}$. A directed edge $(u, v)$ in the potential interference graph implies that the transmission of router $u$ can cause interference at router $v$. Figure 1 shows a sample potential communication graph with 7 mesh routers. The circles around the routers are drawn with radius $R_{T} / 2$ and hence two intersecting circles implies that the two routers are within the transmission range of each other. Figure 2 shows the corresponding potential interference graph with $R_{I}=1.5 R_{T}$. Since $R_{T} \leq R_{I}$, the potential communication graph is always a subgraph of the potential interference graph.

This interference model is different from the frequently used conflict graph representation of interference in a WMN. In the conflict graph representation, a vertex $v_{i j}$ is in the vertex set of the conflict graph if $(i, j)$ is an edge in the potential communication graph. There exists an edge between two vertices $v_{i j}$ and $v_{k l}$ in the conflict graph if min $(\operatorname{dist}(i, k), \operatorname{dist}(i, l), \operatorname{dist}(j, k), \operatorname{dist}(j, l))$

\footnotetext{
${ }^{1}$ Although we consider the standard 802.11 protocol without RTS/CTS, our techniques can be applied to RTS/CTS 802.11 environments with some minor changes.
} 
$\leq R_{I}$. In the context of the problem explored in this paper, our interference model offers some benefits over the conflict graph model. Firstly, our interference model is more intuitive in the sense that it captures the idea of co-channel interference occurring at the receiving nodes in a WMN. Secondly, it can easily be seen that the conflict graph and the potential interference graph can be derived from each other when the potential communication graph is known. Finally, the potential interference graph modeling makes our problem definition more elegant.

\subsection{Graph Definitions and Notations}

All graphs defined in this paper are directed graphs. We drop the prefix directed and henceforth, a graph implies a directed graph. In this section, we introduce the required graph terminology.

- In-degree of a vertex $v$ in a graph $G$ is the number of arcs coming into $v$ and the out-degree of $v$ is the number of arcs going out of $v$. The in-degree and out-degree of a vertex are represented by $\delta_{G}^{-}(v)$ and $\delta_{G}^{+}(v)$ respectively.

- The maximum in-degree of a graph $G$ represented by $\Delta^{-}(G)$ is the maximum of the in-degrees of its vertices. That is, $\Delta^{-}(G)=\max _{v \in V(G)} \delta_{G}^{-}(v)$. Similarly, the maximum out-degree of a graph $G$ represented by $\Delta^{+}(G)$ is the maximum of the out-degrees of its vertices. That is, $\Delta^{+}(G)=\max _{v \in V(G)}$ $\delta_{G}^{+}(v)$.

- A vertex $u$ is a neighbor of vertex $v$ if there is a directed edge from $v$ to $u$. The (closed) neighborhood of vertex $v$ in graph $G(V, E)$ denoted by $N[v]$ is the set that includes $v$ and the neighbors of $v$. The (closed) neighborhood of a subset $S \subseteq V$ of graph $G(V, E)$ denoted by $N[S]$ is the set that includes $S$ and the neighbors of $S$. That is, $N[S]=S \cup \bigcup_{v \in S} N[v]$.

- The subgraph weakly induced by vertex set $S \subseteq V$ in graph $G(V, E)$ is defined as the graph with the vertex set $N[S]$ and edge set $E \cap(S \times N[S])$. In other words, the edge set of the subgraph weakly induced by $S$ consists of all the edges induced by the vertices of $S$ along with the directed edges from set $S$ to its neighbors in the graph. The weakly induced subgraph of graph $G$ on the vertex set $S$ is denoted by $\langle S\rangle_{G}$. An example of the subgraph weakly induced by the set of vertices $S=\{3,6,7\}$ on the potential interference graph of figure 2 is shown in figure 3 .

Throughout this paper, we use the notation $G(V, E)$ to represent the potential communication graph and $H(V, F)$ to represent the potential interference graph.

\subsection{Problem Definition}

The maximum data rate that can be achieved in a multicast structure is limited by the data rate of the bottleneck link. The data rate of the bottleneck link is determined by the amount of interference experienced by the receiver of the link. For instance, a mesh node present in the communication range of 5 transmissions 
experiences more interference and thus provides less throughput compared to a mesh node present in the communication range of 4 transmissions. In an effort towards maximizing the data rate of the multicast structure, we try to select the forwarding group nodes that induce least amount of interference. That is, given the locations of wireless mesh routers, a multicast source node and a set of multicast receivers, our goal is to find the group of forwarding nodes that induce the least interference on the forwarding nodes and the receivers. In terms of the potential communication graph and the potential interference graph, the problem is formally stated as follows.

Given a potential communication graph $G(V, E)$, its corresponding interference graph $H(V, F)$, a multicast source vertex $s \in V$ and a set of receiver vertices $R \subseteq V \backslash s$, find vertex set $S \subset V$ such that

1. the subgraph of $G$ weakly induced by $S$, i.e., $\langle S\rangle_{G}$ has directed paths connecting $s$ to each $r_{i} \in R$ and

2. the maximum in-degree of the vertices of the subgraph of $H$ weakly induced by $S$, i.e., $\Delta^{-}\left(\langle S\rangle_{H}\right)$ is minimized.

We term this problem as the Minimum-Degree Weakly Induced Connected Subgraph (MDWICS) problem. The optimal subset $S$ of the MDWICS problem contains the source vertex and the forwarding group vertices that result in the least interference. In the next section, we prove the hardness of the MDWICS problem.

\section{Computational Complexity}

To prove the NP-completeness of the MDWICS problem, we provide a polynomial time transformation from Exact Cover by 3 Sets (X3C) problem [2]. Consider the scenario in which the interference and transmission ranges of the transmitters are equal. In this case, the potential communication graph and the potential interference graph have the same set of vertices, edges and thus, can be represented by a single graph, say $G(V, E)$. The decision version of the MDWICS problem is then stated as follows.

INSTANCE: Directed graph $G=(V, E)$, vertex $s \in V$ designated as source, vertices $R \subseteq V \backslash s$ designated as the set of receivers and a positive integer $K$.

QUESTION: Is there a subset $S \subset V$ such that the subgraph weakly induced by $S$ in $G$ denoted by $\langle S\rangle_{G}$ has paths from $s$ to each $r_{i} \in R$ and $\Delta^{-}\left(\langle S\rangle_{G}\right)$ is at most $K$.

Theorem 1. MDWICS problem is NP-complete.

Proof. Clearly MDWICS problem is in NP since a nondeterministic algorithm need only guess the vertex set $S$ and check in polynomial time whether the subgraph weakly induced by $S$ in $G$ has paths from $s$ to each $r_{i} \in R$ and the maximum in-degree of the subgraph is at most $K$.

Suppose a finite set $X=\left\{x_{1}, x_{2}, \ldots, x_{3 q}\right\}$ and a collection $\mathcal{C}=\left\{C_{1}, C_{2}\right.$, $\left.\ldots, C_{m}\right\}$ of 3 -element subsets of $X$ make up the instance of X3C. From this 
instance, we will construct an instance of the MDWICS problem using local replacement technique. Corresponding to every element $x_{i} \in X, 1 \leq i \leq 3 q$ and subset $C_{j} \in \mathcal{C}, 1 \leq j \leq m$, introduce vertices $x_{i}$ and $c_{i}$ respectively in graph $G(V, E)$. These vertices together with an additional vertex $s$ make up the vertex set of $G$. The edge set of $G$ consists of two types of edges. The first set includes directed edges $\left(c_{l}, x_{i}\right),\left(c_{l}, x_{j}\right)$ and $\left(c_{l}, x_{k}\right)$ for every subset $C_{l}=\left\{x_{i}, x_{j}, x_{k}\right\}$. The second set includes directed edges from $s$ to each $c_{i}$. The graph constructed using this mechanism has $m+3 q+1$ vertices and $4 m$ edges. Designate vertex $s$ as source and vertices $R=\cup_{i=1}^{3 q} x_{i}$ as the set of receivers. This completes the construction procedure of the proof. Suppose that $K=1$. We claim that there exists a $S \subset V$ such that $\langle S\rangle_{G}$ has paths from $s$ to each $x_{i}, 1 \leq i \leq 3 q$ and $\Delta^{-}\left(\langle S\rangle_{G}\right) \leq 1$ if and only if the corresponding X3C instance contains an exact cover for $X$.

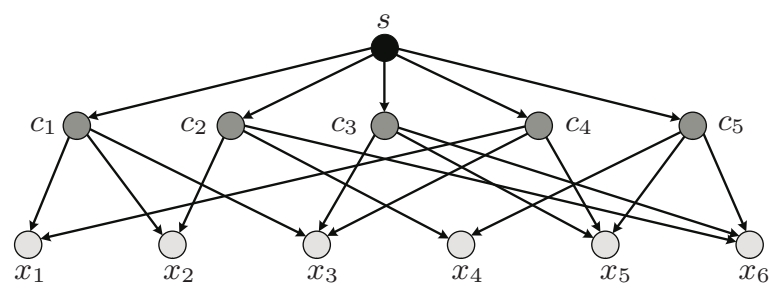

Fig. 4. Local replacement for subsets $C_{1}=\left\{x_{1}, x_{2}, x_{3}\right\}, C_{2}=\left\{x_{2}, x_{4}, x_{6}\right\}, C_{3}=\left\{x_{3}, x_{5}\right.$, $\left.x_{6}\right\}, C_{4}=\left\{x_{1}, x_{3}, x_{5}\right\}, C_{5}=\left\{x_{4}, x_{5}, x_{6}\right\}$

It is easy to verify that if $\mathrm{X} 3 \mathrm{C}$ has an exact cover, then the vertices corresponding to the subsets in the $\mathrm{X} 3 \mathrm{C}$ solution along with vertex $s$ is a solution to the MDWICS problem. Conversely, suppose $S \in V$ such that $\langle S\rangle_{G}$ has paths from source $s$ to each of the receivers $x_{i}^{\prime} s$ and maximum in-degree of $\langle S\rangle_{G}$ is at most 1. Consider the set $S^{\prime}=S \cap\left\{c_{1}, \ldots, c_{m}\right\}$. Note that each vertex $c_{i} \in V$, $1 \leq i \leq m$ has exactly 3 outgoing edges. $\left|S^{\prime}\right|=q$, since otherwise there would be either no path $\left(\left|S^{\prime}\right|<q\right)$ or multiple paths $\left(\left|S^{\prime}\right|>q\right)$ to some $x_{i}$ thereby violating maximum in-degree of 1 . It follows that the subsets corresponding to the vertices in $S^{\prime}$ form an exact cover to the X3C problem.

\section{Optimal Solution}

In this section, we provide an Integer Linear Program (ILP) formulation 3] to solve the MDWICS problem optimally. Given a potential communication graph $G(V, E)$, potential interference graph $H(V, F)$, source $s \in V$ and receivers $R \subseteq$ $V \backslash s$, the problem is to find subset $S \in V$ that minimizes the maximum in-degree of $\langle S\rangle_{H}$ subject to the constraint that $\langle S\rangle_{G}$ has paths from $s$ to each $r_{i} \in R$. The ILP finds the weakly induced subgraph that achieves the above mentioned goal. From this subgraph, we can extract the desired node set $S$ by removing leaf nodes, that is, nodes with zero out-degree. 
The indicator variables are defined as follows. $x_{i, j}=1$, if edge $(i, j) \in E$ is in the optimal solution, 0 otherwise. Define $f_{i, j}^{p}=1$, if there is a flow from $s$ to receiver $p$ through link $(i, j) \in E$ in the optimal solution, 0 otherwise. $f_{i, j}^{p}$ is used to ensure connectivity from $s$ to receiver $r$. Define $y_{i, j}=1$ for edge $(i, j) \in F$ if node $i$ is the transmitter in the optimal solution, 0 otherwise. $y_{i, j}$ is used to capture the interference caused by $i$ 's transmission on node $j$. The objective is to minimize $D$, the maximum in-degree of all the nodes in the interference subgraph. The following set of constraints define the problem accurately.

- The degree constraint specifies that the maximum in-degree of the nodes in the optimal solution interference subgraph should be no larger than $D$. That is, $\sum_{(i, v) \in F} y_{i, v} \leq D, \forall v \in V$.

- The broadcast constraint captures the broadcast characteristics of the wireless medium. When a node transmits, its transmission affects all nodes in its transmission range. This is represented by $x_{(v, i)}=x_{(v, j)}, \forall v \in V,(v, i)$ and $(v, j) \in E, i \neq j$.

- The interference constraint models the interference characteristic of the network. For every node in the potential communication graph, if there is a directed communication edge going out, then there is a directed interfering edge going out from the corresponding node in the potential interference graph. This is represented by $y_{v, i} \geq x_{v, j}, \forall(v, i) \in F, \forall(v, j) \in E$.

Connectivity from the source node to each receiver is ensured by the following flow conservation constraints. These constraints are similar to the multi-commodity flow constraints 3 .

- The total incoming flow into an intermediate node is equal to the total outgoing flow from that node. That is, $\sum_{(i, v) \in E} f_{i, v}^{p}=\sum_{(v, j) \in E} f_{v, j}^{p}, \forall p \in$ $[1, k], \forall v \in\{V \backslash\{s \cup R\}\}$.

- For each receiver and for each flow, the incoming flow is equal to outgoing flow except for the flow destined for the receiver. $\sum_{(i, r) \in E} f_{i, r}^{p}=\sum_{(r, j) \in E}$ $f_{r, j}^{p}, \forall p \in\{R \backslash r\}, \forall r \in\{R\}$.

- There is zero incoming flow and unit outgoing flow to the source node. That is, $\sum_{p=1}^{|R|} \sum_{(i, s) \in E} f_{(i, s)}^{p}=0$ and $\sum_{(s, i) \in E} f_{s, i}^{p}=1, \forall p \in R$

- For each receiver, the outgoing flow from that receiver should be zero, if the flow is for the same receiver. $\sum_{(r, j) \in E} f_{r, j}^{r}=0, \forall r \in R$

- The dependence between $f_{i, j}^{p}$ and $x_{i, j}$ for edge $(i, j) \in E$ is represented by $|R| \times x_{i, j} \geq \sum_{p=1}^{|R|} f_{i, j}^{p}, \forall(i, j) \in E$

\section{Proposed Algorithms}

In this section, we first present a centralized heuristic for the MDWICS problem and then describe the design of a distributed version.

\subsection{Centralized Heuristic}

The greedy heuristic takes as input the potential communication graph $G(V, E)$, the potential interference graph $H(V, F)$, multicast source vertex $s$ and multicast 
receiver set $R$. The output of the heuristic is the set of multicast forwarding group nodes. In the algorithm, $\langle S\rangle_{G}$ and $\langle S\rangle_{H}$ are the weakly induced subgraphs of $G$ and $H$ respectively on vertices $S$ as defined before. The algorithm maintains a feasible solution set $S$ of vertices that contain paths from the source vertex to all the receivers and a set $W$ of vertices that contains the set of visited vertices. Initially, all vertices in the graph are included in set $S$ and set $W$ contains the source vertex (Step 1). The algorithm then selects in each iteration a vertex in $S \backslash s$ that has the maximum in-degree in the subgraph weakly induced by $S$ in graph $H$. If there are multiple such vertices, any one of them is selected arbitrarily. This vertex will be a potential candidate for removal since it has the highest in-degree in $\langle S\rangle_{H}$. Step 5 checks if removal of this vertex from the graph $G$ disconnects any of the receivers from $s$. If not, this vertex and all its incident edges are removed from both $G$ and $H$. This vertex is added to the set of vertices $W$ visited so far. The algorithm terminates when all vertices in the graph have been visited.

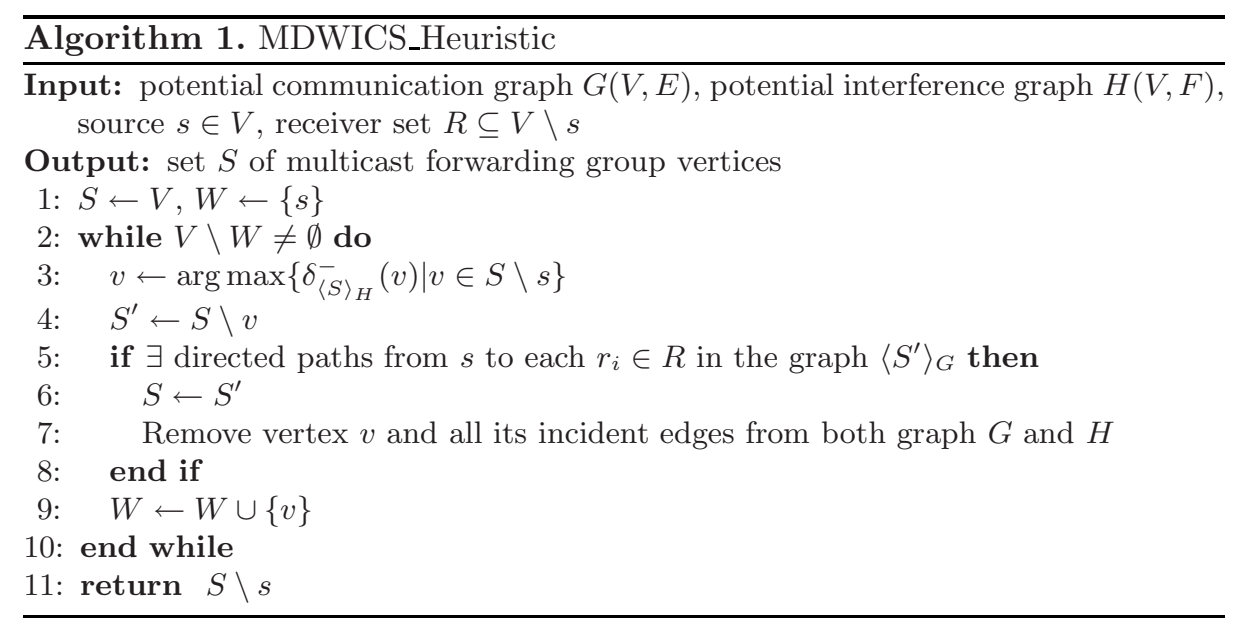

\subsection{Distributed Protocol}

The design of the distributed interference-aware multicast protocol is based on the Optimized Link State Routing (OLSR) protocol 4. OLSR could be used in mesh networks to maintain the state and quality of the links. The distributed protocol takes full benefit of the topology knowledge obtained by the OLSR protocol with its Topology Control (TC) messages. The TC messages are efficiently dispersed in the network through multipoint relays (MPRs). Setting the parameter TC_REDUNDANCY $=2$ at each node in the OLSR protocol ensures that each node gets information about every other node, link in the network (Section 15.1 [4]). Each node in the network is uniquely identified by its IP address. The source initiating the multicast session generates a unique ID for the session based on its IP address and a sequence number for the multicast group. 
The source with its topology information independently computes the set of multicast forwarding group nodes. It then disseminates through the MPRs, a MC_FG message consisting of the IP addresses of the forwarding group nodes and a MC_JOIN message to the multicast group members. A node upon receiving a MC_FG message checks if its IP address is listed in the message. If so, it records the session ID in a forwarding group (FG) table. Only the first MC_FG message received by a node is broadcasted with subsequent MC_FG messages being discarded. The MC_JOIN message is to inform the multicast group members about the initiation of the session. The session ID is included in every packet forwarded by the multicast source. If a node receiving this packet has the session ID listed in its FG table, it forwards the packet. Evaluation of this distributed version is the focus of current ongoing work.

\section{Simulation Environment, Results and Discussion}

We conducted extensive experiments to evaluate our centralized heuristic. We compared the performance of our heuristic with the Shortest Path Tree (SPT) algorithm, Minimum Steiner Tree (MST) approximation algorithm [5] and the optimal solution obtained by solving the ILP formulation given in section 4 The Shortest Path Tree algorithm finds the set of edges connecting $s$ to each receiver such that the length of the shortest path (measured as the number of hops) from $s$ to each receiver is minimized. The MST algorithm presented in [5] is a $O\left(\log ^{2} k\right)$-approximation algorithm ( $k$ is the number of receivers) for the Minimum Steiner Tree problem. We used Cplex 10 to solve the ILPs for the optimal solution.

In all our experiments, the number of mesh routers in the network was fixed to be 70 . The area for the deployment of the mesh nodes was a square area whose sides were computed based on the required node-density. For instance, for a required node-density of 100 nodes $/ \mathrm{km}^{2}$, the locations of the 70 nodes were randomly generated in a square of area of $0.7 \mathrm{~km}^{2}$. The locations of the 70 nodes were generated randomly. The transmission radius of the mesh routers was fixed at $100 \mathrm{~m}$. The multicast source and the multicast receivers were selected randomly. The simulation scenarios were designed to measure the impact of three parameters namely, the number of receivers, the density of the network (nodes per unit-area) and the ratio of interference radius to transmission radius $\left(R_{I} / R_{T}\right)$ on the interference-degree of the multicasting structure produced by the four approaches. The interference-degree is the maximum number of forwarding group mesh nodes that affect any node in the multicast structure. As discussed before, this determines the maximum achievable data rate for the multicast structure.

In the first set of experiments (figure 5), the number of receivers was fixed at 28 and the node-density at 100 nodes $/ \mathrm{km}^{2} . R_{I}$ was increased from $100 \mathrm{~m}$ to $250 \mathrm{~m}$ in steps of $50 \mathrm{~m}$ resulting in $R_{I} / R_{T}$ ratio to vary between 1 and 2.5 . It is to be noted from figure 5 that as the $R_{I} / R_{T}$ ratio, in turn the interference range of the mesh routers increase, the interference-degree increases rapidly. This is 
intuitive since an increase in the interference range introduces more interference on the mesh nodes. For all values of $R_{I} / R_{T}$ ratio, our heuristic performs better than the SPT and MST algorithms.

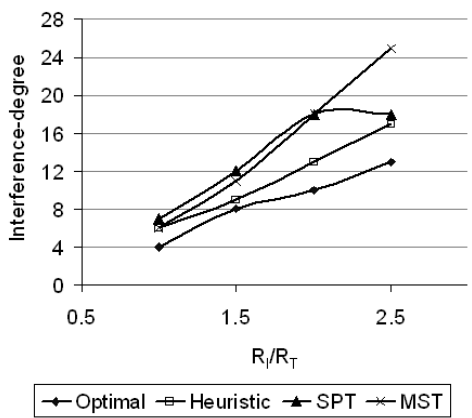

Fig. 5. Interference-degree vs $R_{I} / R_{T}$ ratio with \# Receivers $=28$ and nodedensity $=100$ nodes $/ \mathrm{km}^{2}$

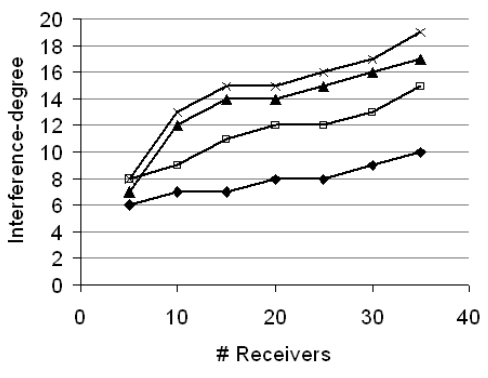

Optimal $\leftarrow$ Heuristic $₫$ SPT $*$ MST

Fig. 6. Interference-degree vs \# receivers with $R_{I} / R_{T}=2$ and nodedensity $=100$ nodes $/ \mathrm{km}^{2}$

In the second set of experiments (figures 6, 7), we fixed the $R_{I} / R_{T}$ ratio at 2 . For node densities 100 nodes $/ \mathrm{km}^{2}$ and 200 nodes $/ \mathrm{km}^{2}$, we plotted the variation of the interference-degree with the number of receivers. It can be observed that as the number of receivers increases, the interference-degree for each algorithm increases. However, the interference-degree of the heuristic stays closer to the optimal compared with the SPT and MST algorithms.

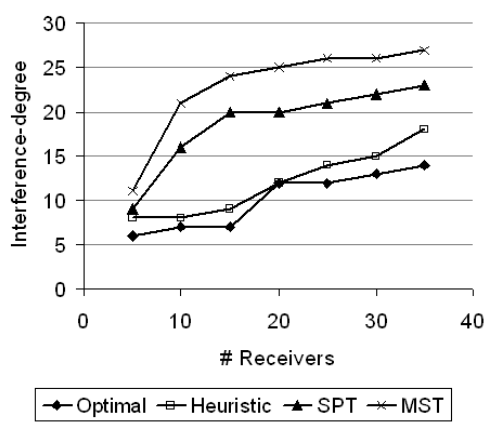

Fig. 7. Interference-degree vs \# receivers with $R_{I} / R_{T}=2$ and nodedensity $=200$ nodes $/ \mathrm{km}^{2}$

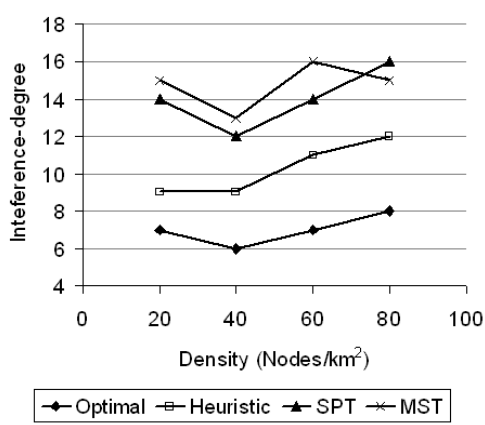

Fig. 8. Interference-degree vs nodedensity with $R_{I} / R_{T}=1$ and \# receivers $=28$

In the next experiments (figure 8), we studied the effect of varying density on the interference-degree. The number of receivers was set at 28 and the $R_{I} / R_{T}$ 
ratio at 1.5 . The results may seem to be counter-intuitive at first glance, since increased density must lead to increased interference-degree and the first two points in the graph do not confirm this observation. However for each value of node-density, the area in which the nodes are deployed changes and thus, the locations of the nodes are recomputed for each node-density resulting in different communication and interference topologies.

In all the experiments, the performance of our heuristic matches closely with that of the optimal. The performance SPT and MST algorithms in most of the cases are far from optimal. This is natural since these algorithms prefer shorter paths from source to the receivers and do not pay attention to the interference caused by these paths on the network nodes.

\section{Related Work}

Most of the related work in this area focusses on efficient network layer multicast and broadcast in multihop wireless networks and MANETs. Multicast schemes can be classified as either source-based or mesh-based. Source-based protocols construct shortest paths from source to the receivers. AMRIS, MAODV and MOLSR [6] are the well known source-based protocols. The mesh-based protocols consider multiple paths from source to the receivers. Examples of meshbased protocols are ODMRP, CAMP and FGMP [7. In the presence of mobility, mesh-based protocols are advantageous as they maintain alternate paths for each receiver. Another scheme [8] based on on-demand routing and genetic algorithm executes faster than the conventional multicast schemes. Moreover, 802.11 QoS issues studied in 9] reveal that the design framework of multicast protocols should share flow characteristics across multiple layers and cooperate to meet the application's requirement. Study done in [10] states that in multicasting there is no one size fits all protocol that can optimally serve the need of all types of application. Recently, a joint optimization approach in [1] emphasizes network coding technique for multicast routing and game theory approach for interference management. The closest work to ours is [12] in which the problem of computing multicast trees with minimal bandwidth consumption in mesh networks is considered. The authors show that this NP-complete problem is equivalent to minimizing the number of multicast transmissions rather than the edge cost or the total number of edges of the multicast tree. On the contrary, a tree with minimum number of transmissions may not provide minimum interference or maximum bandwidth.

\section{Conclusion and Future work}

In this paper, we categorized the interference-aware multicasting problem as a graph problem of finding a weakly induced subgraph of minimum degree. For this purpose, we introduced a new model of interference called the potential interference graph. We analyzed the intractable nature of this problem and presented an efficient greedy-based heuristic algorithm. We also presented a distributed 
extension of our heuristic. The simulation results provide substantial evidence of the superior performance of our heuristic compared to the Shortest Path Tree and Minimum Steiner Tree approximation algorithms. Future work in this area lies in analyzing the performance of the distributed algorithm and providing approximation bounds to the centralized algorithm.

\section{References}

1. Online: City-wide wi-fi projects. (http://www.seattlewireless.net http:// Www.wirelessphiladelphia.org, http://www.waztempe.com)

2. Garey, M., Johnson, D.: Computers and Intractability: A Guide to the Theory of NP-Completeness. Freeman Press (1979)

3. Bazaraa, M., Jarvis, J., Sherali, H.: Linear Programming and Networks Flows. John Wiley \& Sons (2004)

4. Online: Rfc 3626 - optimized link state routing protocol (olsr). (http://www . ietf.org/rfc/rfc3626.txt)

5. Charikar, M., Chekuri, C., yat Cheung, T., Dai, Z., Goel, A., Guha, S.: Approximation algorithms for directed steiner problems. In: ACM-SIAM Symposium on Discrete Algorithms (SODA). (1998)

6. Kunz, T., Cheng, E.: Multicasting in ad-hoc networks: Comparing maodv and odmrp. In: International Conference on Distributed Computing Systems (ICDCS). (2002)

7. Madruga, E., Garcia-Luna-Aceves, J.: Multicasting along meshes in ad-hoc networks. In: International Conference on Communications. (1999)

8. Banerjee, N., Das, S.: Modern: Multicast on-demand qos-based routing in wireless networks. In: Vehicular Technology Conference. (2001)

9. Zhu, H., Li, M., Chlamtac, I., Prabhakaran, B.: A survey of quality of service in ieee 802.11 networks. IEEE Wireless Communications 11 (2004)

10. Gossain, H., Cordeiro, C., Agrawal, D.: Multicast: wired to wireless. IEEE Communications Magazine 40 (2002) 116-123

11. Yuan, J., Li, Z., Yu, W., Li, B.: Cross-layer optimization framework for multihop multicast in wireless mesh networks. IEEE Journal on Selected Areas in Communication 24 (2006)

12. Ruiz, P., Gomez-Skarmeta, A.: Heuristic algorithms for minimum bandwith consumption multicast routing in wireless mesh networks. In: ADHOC-NOW. (2005) 